\title{
La Maladie de Sachs : le réel construit entre les pôles opposés du savoir et de la subjectivité
}

\author{
Christian Milat \\ Université d'Ottawa
}

Sous le pseudonyme perecquien de Martin Winckler, le docteur Marc Zaffran est, en tant qu' « écrivant», l'auteur, seul ou en collaboration, de plusieurs ouvrages médicaux et d'essais sur la relation soignant-soigné. Il possède en outre un site Web où figurent, outre la reproduction d'articles, de nombreuses informations médicales, notamment sur la contraception. Comme "écrivain », il a en particulier publié plusieurs romans, dont un cycle de trois textes orientés autour du personnage d'un médecin, Bruno Sachs : La Vacation, La Maladie de Sachs et 
Les Trois Médecins. Le fait que ces trois fictions habitent un univers médical et qu'elles sont l'œuvre d'un médecin laissent à penser que leur référent a toutes les chances de se rapporter au réel, que l'auteur est censé bien connaître. Du reste, dans la présentation que, sur son site Web, P.O.L fait de La Maladie de Sachs, il est mentionné qu'il s'agit d'un « document sur l'état de la médecine en France aujourd'hui » et que

Martin Winckler, qui connaît la question de très près, va tenter cette gageure d'une description qui serait aussi une proposition de thérapie. Décrire tout à la fois le quotidien des patients et celui du médecin, dans le même mouvement, au même rythme, comprendre celui qui soigne comme lui-même comprend ses malades au point de vivre leurs souffrances ${ }^{1}$.

La dimension autoreprésentative de ce roman ajoute à cette promesse de réalisme. En effet, considéré, quand il était enfant, comme « un littéraire $»^{2}$, le docteur Sachs écrit « un livre [...] un roman » $(M S$, p. 177) qui constitue, à ses yeux, « un livre sur [s]on expérience de médecin». Dans la salle d'attente du même docteur Sachs, ce roman est lu par l'un des personnages (voir MS, p. 14 et passim). À la fin de La Maladie de Sachs, ce lecteur patient - avec les deux acceptions de ce vocable apostrophe le médecin en ces termes : «Je sais qu'il y a un autre nom sur la plaque, mais vous êtes Martin Winckler, n'est-ce pas? C'est bien vous qui avez écrit La Maladie de Sachs? » (MS, p. 658) S'il est le praticien qui se cache sous le pseudonyme de Martin Winckler, le docteur Sachs, nécessairement, n'est autre que Marc Zaffran, dont le roman constituerait en quelque sorte

1 P.O.L, http://www.pol-editeur.fr/catalogue/fichelivre.asp?Clef=298, site visité le 30 avril 2007.

2 Winckler, 2005, p. 176. Désormais, les citations de cet ouvrage seront suivies, entre parenthèses, d'un renvoi aux pages de cette édition, précédé du sigle $M S$. 
le témoignage. Au-delà de cette composante réflexive, la présente étude se propose d'examiner, dans La Maladie de Sachs, les différents processus de production d'un réel qui s'affiche d'emblée doté de nombreux gages d'authenticité, mais qui apparaît finalement comme une construction largement subjective.

\section{L'exhibition de preuves d'authenticité}

Plusieurs éléments témoignent de la préoccupation de l'auteur en matière de réel. En effet, dans un "Avertissement", s'il est signalé que "tous les personnages de ce roman sont fictifs » ( $M S$, p. 11), il est immédiatement ajouté que «les événements décrits dans ces pages semblent plus vrais que nature » et qu'au surplus, «même lorsqu'elles ne sont pas délibérées, les ressemblances avec des personnes ou des événements réels sont, probablement, inévitables ». Ainsi, dès avant l'incipit, le lecteur est informé des tensions qui existent, au sein d'un texte qui est au demeurant rattaché au genre romanesque, entre ce qui relève de l'imagination et ce qui a trait au réel, celui-ci constituant le référent à partir duquel celle-là a pu s'exercer. Or, à cette mention liminaire fait écho celle qui figure cette fois après la fin du roman. Dans un «Post-scriptum», l'auteur reprend en effet la parole : «Je tiens à témoigner ma gratitude aux habitants de "Play" et du "canton de Lavallée". » (MS, p. 661) Sans doute faut-il voir, derrière ces deux toponymes fictifs, le canton de Ballon ainsi que le village de Joué-L'Abbé, où Marc Zaffran a ouvert en 1983 un cabinet de médecine générale près du Mans, dans la Sarthe. Ces habitants ont, souligne Martin Winckler, «écrit ce livre avec moi ». Tous apparaissent ainsi comme les garants du respect que le roman accorderait au réel. 
Or, à ces cautions anonymes s'ajoutent celles de 139 personnes qui, désignées par leurs prénoms suivis d'une initiale, apparaissent comme autant d'auxiliaires et qui, à ce titre, apportent chacune à l'œuvre une parcelle de réel : « Je voudrais enfin dire toute mon affection pour celles et ceux qui, de très près ou de très loin, m’ont accompagné et soutenu au cours des cinq années écoulées » (MS, p. 661 et 662).

Dans le même "Post-scriptum », l'auteur manifeste une fois de plus le souci qu'il nourrit à l'égard du réel. En effet, dans le début du roman (voir $M S$, p. 17) est insérée la reproduction du Serment d'Hippocrate, ce qui constitue l'irruption, au sein même de la fiction, d'un fragment arraché au réel. Or, l'auteur est à ce point sourcilleux sur l'authenticité de cette pièce qu'il s'attache à informer son lecteur qui connaîtrait le texte exact du serment - et qui observerait, entre celui-ci et la reproduction que le roman en fait, une différence - qu'il en « existe plusieurs versions » $(M S$, p. 661) et que celle qui est reproduite « apparaît en page 3 d'une thèse de médecine imprimée à Alger en 1939 -. Elle est sensiblement différente du texte grec originel. » Ainsi est affirmée la véracité du texte reproduit, même si la teneur de l'attestation peut éveiller des doutes chez un lecteur averti.

Le roman reproduit plusieurs autres documents réels, visant par là à renforcer l'illusion référentielle. Par exemple, lorsque le docteur Sachs s'apprête à remplir une feuille de sécurité sociale, la voix narrative, au lieu d'énoncer les informations que le médecin y inscrit, c'est-à-dire au lieu de formuler des données personnelles, reproduit la feuille officielle, celle qui était en vigueur en 1998, et ce, de façon fidèle, en restituant les numéros, placés en gras ou en maigre, 
qui structurent les différentes rubriques, et en respectant scrupuleusement la casse des mots : certains sont composés en capitales, d'autres en petites capitales ou en bas-de-casse. Ainsi, le lecteur a sous les yeux ce que le docteur Sachs, comme tout véritable médecin de l'époque, peut observer :

la case des NOM ET PRÉNOM DU MALADE (à remplir par le médecin selon les indications de l'intéressé), puis les rubriques surmontées des mots PRESTATION DES ACTES : 1 - date des actes médicaux; 2 - désignation des actes suivant nomenclature; 3 - délivrance d'une ordonnance; 4 prescription: 1 (chambre) ou 2 (sortie autorisée) [...]. (MS, p. 31)

Le réel s'invite de la même manière dans la fiction aux deux pages (MS, p. 652 et 653 ) de la section no 112 . Dépourvue de tout élément diégétique - son titre, "ÇA FINIT COMME ÇA », mis à part —, cette section est entièrement constituée de la transcription, exacte, du certificat de décès de l'époque :

À remplir par le Médecin

Commune :

Le Docteur en médecine soussigné, certifie que la mort de la personne désignée ci-contre, survenue le... à.... heures, est réelle et constante.

nom (Obstacle médico-légal à l'inhumation oui/non), prénoms (Don du corps oui/non), âge (Obligation de mise immédiate en cercueil hermétique oui/non), sexe (Obligation de mise immédiate en cercueil simple oui/non), domicile. [...] (MS, p. 652)

Là encore, sont scrupuleusement respectées la casse, la graisse et la face (italique ou romain) des caractères. Le certificat reproduit est conforme au certificat réel à un détail près, qui n'apparaît que moyennant une lecture très attentive: aux exemples de causes de décès par homicide, «I. a) Section de 
l'artère fémorale, b) Homicide par coup de couteau » (MS, p. 653), l'auteur a ajouté, non sans humour : «Conflit familial $»^{3}$. Cet ajout invalide bien évidemment l'authenticité du document censément reproduit, mais en même temps, du fait de sa pertinence, ne remet pas en cause le réalisme de l'énoncé qui, bien au contraire, semble effectivement "plus vra[i] que nature » $(M S$, p. 11).

Une autre section, la section $\mathrm{n}^{0} \mathrm{81}$, est entièrement consacrée à un texte qui semble être tiré d'un ouvrage de médecine. Deux pages, en effet, sont occupées par l'énumération des symptômes, des signes cliniques par lesquels le cancer se manifeste et qui sont formulés au moyen de termes hautement spécialisés :

Parmi les signes secondaires à la croissance parenchymateuse ou endobronchique de la tumeur primitive figurent la toux, des hémoptysies, un wheezing et un stridor, une dyspnée ou une pneumopathie (avec fièvre et toux expectorante), résultant de l'obstruction des voies respiratoires... (MS, p. 457)

L'illusion réaliste est accentuée par le renvoi à ce qui apparaît comme l'entrée d'un dictionnaire médical: «Des métastases extrathoraciques (voir ce terme) sont découvertes à l'autopsie dans plus de cinquante pour cent des épithéliomas épidermoïdes, quatre-vingts pour cent des adénocarcinomes. » En fait, s'il n'a pas reproduit telle quelle la page d'un ouvrage, l'auteur s'est néanmoins inspiré de plusieurs traités ${ }^{4}$, dont il a tiré en quelque sorte la synthèse. Ici, même si la section n'exprime aucun lien explicite avec la diégèse, le lecteur sait que la présence de ces informations se justifie par la maladie dont souffre l'un des « narrateurs », Monsieur Guilloux (voir MS,

3 C'est M. Winckler qui nous a signalé cet ajout (courriel du $1^{\mathrm{er}}$ avril 2007).

4 L'information nous a été fournie par l'auteur (courriel du $1^{\mathrm{er}}$ avril 2007). 
p. 201). En renforçant l'ethos du romancier, l'appel au savoir médical ancre la fiction dans le réel: le lecteur possède les preuves que le texte émane d'un écrivain qui connaît d'expérience le sujet dont il traite, ce qui lui donne la conviction que le contenu de la fiction est conforme à la réalité.

Même si, parmi les quelque 650 pages du roman, les interventions du docteur Sachs sont largement minoritaires elles ne couvrent qu'environ 85 pages -, elles jouent néanmoins un rôle important dans la production de l'effet de réel. N'intervenant pratiquement jamais en tant que narrateur, le médecin ne participe guère directement à la fiction, mais il contribue efficacement à enraciner celle-ci dans la réalité puisque, à plusieurs reprises, ce sont des documents rédigés de sa main qui sont reproduits. Au surplus, ces textes ont toujours rapport avec la médecine. Ainsi, la nouvelle dont le docteur Sachs est invité à relire les épreuves, intitulée «CABINET PORTRAIT » (MS, p.607), éloigne certes la diégèse du cabinet médical pour la diriger vers les cabinets, mais elle comporte néanmoins la relation d'une maladie, l'« invagination intestinale aiguë» (MS, p. 613). La communication donnée par le docteur Sachs à un "Colloque Littérature et Médecine [organisé à] Tourmens » (voir $M S$, p. 643-649), insère, une fois encore, au sein de la fiction, un texte dont la teneur tend à rapprocher celle-ci du réel.

Il en va de même des textes que le docteur Sachs est censé avoir rédigés dans des « cahiers » $(M S$, p. 133, voir p. 375, 399 et 586) ou des carnets (voir $M S, 397)$. Dans tous les cas, ces « pages manuscrites » (MS, p. 387), ces «feuillets détachés d'un grand bloc » $(M S, 462)$, sont « des observations, des rapports, des dossiers » (MS, p. 509). Ce sont des écrits réels qui portent sur 
des faits réels. Ce sont des fragments issus directement de l'expérience d'un médecin. Ainsi, la section no 72 du roman est totalement constituée de la retranscription de notes que le docteur Sachs a prises de façon succincte à propos de patients suivis d'ordinaire par des confrères et qui sont destinées à informer ceux-ci des problèmes rencontrés par leurs malades et des soins qui leur ont été prodigués :

Visites dimanche :

Jules Gavarry, Deuxmonts (Dr Boulle), sciatique - dans la nuit de samedi à dimanche, je lui ai fait des anti-inflammatoires IM [...]

Armand Duras, Sainte-Sophie (Dr Jardin), poussée insuff. cardiaque $[\ldots]$

Arnaud Belleto, Marquay (Dr Boulle), grippe

Janine Daudet, Saint-Jacques (Dr Jardin), certificat de décès (MS, p. 397)

Si la contribution directe du docteur Sachs au roman est limitée, c'est que ses faits et gestes, ses paroles, ses pensées, ses émotions, sont relatés par de nombreux personnages, qui se conduisent comme autant d'instances narratives. Il s'agit de sa mère, de sa compagne (Pauline), d'un cousin (Roland), d'amis (Catherine, Ray, Diego), de sa femme de ménage (Mme Borgès), de voisins (une boulangère, une tenancière de café). Il s'agit aussi de personnages appartenant au monde médical: sa secrétaire (Mme Leblanc), des pharmaciennes, plusieurs médecins et, en particulier, ses patients, des malades des deux sexes et de tous les âges. Ce procédé polyphonique comporte, lui aussi, une visée réaliste. En effet, plus qu'un narrateur autodiégétique ou hétérodiégétique, de nombreuses voix narratives sont capables de rendre la complexité, la diversité du réel. Cette restitution peut paraître au lecteur d'autant plus objective qu'elle est engendrée par la multiplication des points 
de vue subjectifs de personnages qui sont tous, aux côtés du docteur Sachs, des acteurs ou des témoins.

$\mathrm{Du}$ reste, cette qualité des personnages narrateurs explique la précision de leur narration. C'est qu'eux aussi racontent ce qu'ils ont vécu. D'où la minutie avec laquelle ils détaillent chaque mouvement :

Tu passes devant nous, tu ouvres la porte de communication et tu la retiens d'un coude. De l'autre main, tu isoles une clé du trousseau, tu déverrouilles la seconde porte, tu l'ouvres. Tu ôtes la clé de la serrure, tu glisses le trousseau dans ta poche, tu entres. Silencieusement, la porte de communication se referme derrière toi sous l'action du groom automatique. ( $M S$, p. 19)

Jointe à l'utilisation du présent de l'indicatif, cette relation méticuleuse n'est pas sans rappeler celles qu'on peut lire sous la plume des Nouveaux Romanciers et, conduite à la seconde personne, elle n'est pas sans faire écho au procédé employé par Butor dans La Modification. Compte tenu du soin avec lequel les narrateurs rédigent leurs descriptions, il est naturel que les lieux, et surtout la pièce où le docteur Sachs reçoit ses patients, soient munis de tout l'équipement médical requis : «petit évier» $(M S$, p. 24), « lit bas» (p. 24), «table d'examen à tubulures chromées », «pèse-personne » (p. 28), " pèse-bébé » (p. 24), «autoclave» (p. 269), «appareil à tension» et «son brassard gris » (p. 25), «petit marteau à tête ronde entourée d'un caoutchouc sombre » (p. 35), «abaisse-langue en bois» (p. 27), «distributeur en carton [pour] gants informes en caoutchouc translucide» (p.36), "cahier de rendez-vous» (p. 30), «bloc d'ordonnances» (p. 21), «feuilles de sécurité sociale » (p. 29), etc. Le docteur Sachs lui-même est doté des attributs propres à sa fonction, «sacoche» (p.19) et, en particulier, «blouse blanche » (p.19). Les narrateurs insistent 
sur le rapport étroit qui existe entre ces détails et la réalité du personnage : «C'est bien de mettre une blouse. Ça fait vraiment docteur» (p. 141), surtout que «[s]ur la poche de poitrine, le mot Médecin est bien lisible» (p. 154).

Parmi les procédés destinés à restituer le réel figure enfin l'accumulation d'éléments énumérés sous forme de liste. Due à un narrateur qui pourrait être le docteur Sachs, la section intitulée «LES PLAINTES » (MS, p. 93), par exemple, est tout entière bâtie selon ce schéma :

Qu'est-ce qui ne va pas?

J'ai mal au ventre.

Je perds mes cheveux.

J'ai une verrue.

Je ne vois plus d'un œil.

J'ai la tête qui tourne, ça serait pas la tension?

J'ai mal au dos.

J'ai toujours soif.

J'ai mal au pied.

Ça me gêne de vous le dire mais j'ai une douleur mal placée.

Je peux plus bouger.

Je saigne.

Je n'en peux plus.

J'ai un truc là, dans la bouche. Ça me fait peur. (MS, p. 100)

Écartant toute chronologie, regroupant sous une même rubrique tous les cas de figure correspondants, quels que soient les personnages concernés, l'énumération est ici un moyen d'évoquer de façon authentique la réalité, où se mêlent tout et son contraire :

Pourquoi venez-vous me voir ce soir?

[...]

Parce que j'ai grossi.

Parce que j'ai maigri.

Parce que je ne dors plus.

Parce que je dors sans arrêt. (MS, p. 100 et 101) 
Concentré d'expériences, résumé de vécus, l'écriture-somme se fait inventaire, cherchant dans l'exhaustivité du recensement à saisir le réel dans sa totalité.

\section{Une construction largement subjective}

Au rebours, il existe dans La Maladie de Sachs des indices selon lesquels les énoncés ou les procédés qui semblent participer à la représentation, dans la fiction, du réel, du vrai monde, contribuent bien plutôt à l'élaboration d'un univers différent de celui-ci.

Ainsi, loin d'apporter à la diégèse la garantie du réel, la reproduction d'informations puisées au sein d'ouvrages médicaux lui incorpore au contraire des ingrédients qui lui sont étrangers. En effet, dans la section no 86, intitulée «PREMIÈRES OBSERVATIONS», le docteur Sachs relate notamment ses premières expériences d'étudiant en médecine. Relisant les épreuves d'un traité de médecine dirigé par un de ses professeurs, il imagine que connaître « les symptômes de toutes les affections mortelles [...] [1]'immuniserait contre elles » $(M S$, p. 479). En fait, il n'en est rien : « Bien plus tard, j'ai compris que les maladies des traités ne sont elles-mêmes que le produit d'une systématique arbitraire. Dans la réalité, on ne meurt pas comme dans les livres de médecine.» (MS, p. 479 et 480) Apparaît ainsi un divorce entre, d'une part, le savoir médical, tout théorique, et d'autre part, la vraie vie.

Cette vraie vie, ce sont les témoignages des différents narrateurs qui sont censés en rendre compte. Leurs descriptions, on l'a vu, sont très souvent fort minutieuses. Il arrive qu'elles le soient trop. Ainsi, une patiente raconte : 
Tu prends le stéthoscope, tu ajustes les écouteurs à tes oreilles, tu poses le pavillon à la saignée de mon coude, tu saisis la poire en caoutchouc de l'appareil à tension, tu visses la molette et tu te mets à gonfler. (MS, p. 25)

Cette phrase renferme plusieurs vocables - pavillon, saignée, molette notamment - qui, appartenant à la terminologie de la médecine, offrent peu de probabilités de se trouver dans la bouche d'une simple patiente. De même, ailleurs dans le roman, une autre femme note: "Tu prends une cupule en métal » $(M S$, p. 270). Le même terme, " cupule » (MS, p. 393), est repris plus loin, cette fois par une jeune fille. Dans les deux cas, on comprend mal que ce mot spécialisé puisse faire partie du vocabulaire des personnages, ce qui remet en cause l'authenticité de leur discours. En fait, ces signes sont révélateurs: contaminés par des termes réservés au jargon médical, les énoncés, bien qu'ils apparaissent au lecteur inattentif comme émanant des différentes instances narratives, sont finalement produits par le narrateur qui, lui, maîtrise ce langage : le docteur Sachs.

Si ces énoncés perdent le réalisme attaché à leurs énonciateurs supposés, cette perte est susceptible, semble-t-il, d'être contrebalancée par le réalisme dont peut se prévaloir le docteur Sachs, puisque celui-ci ne ferait, au travers des témoignages de son entourage, que restituer ses expériences passées. En fait, là encore, le réel risque de manquer au rendezvous, et ce, à cause des souvenirs, à la fois incertains et lacunaires :

J'ai vu passer des milliers de personnes, avoue Bruno Sachs, mais en cet instant même, je ne pourrais spontanément en évoquer qu'une douzaine [...]... Alors, je crois qu'écrire, pour un médecin comme pour n'importe qui, c'est prendre la mesure de ce qu'on se ne rappelle pas, de ce qu'on ne retient pas. Écrire, 
c'est tenter de boucher les trous du réel évanescent avec des bouts de ficelle, faire des nœuds dans des voiles transparents en sachant que ça se déchirera ailleurs. Écrire, ça se fait contre la mémoire et non pas avec. Écrire, c'est mesurer la perte. (MS, p. 649)

Les défaillances de la mémoire ouvrent dans le réel une brèche où s'engouffre l'imagination, mère de la fiction.

Par ailleurs, si c'est Bruno Sachs qui assure globalement la prise en charge réelle de la narration, il est normal que le réel produit dans La Maladie de Sachs porte sa marque, étant entendu que, comme l'ont notamment précisé les membres de l'École de Palo Alto, le réel est toujours lié à la représentation que s'en fait un individu (voir Watzlawick, 1975). Dans ces conditions, il est possible de faire du point de vue du docteur Sachs une explication au tableau très manichéen qui est brossé dans l'ensemble du roman et dont la stricte conformité au réel peut, de ce fait, être suspectée. En effet, en dehors des quelques docteurs nommément désignés dans le roman qui, eux, font souvent l'objet de commentaires positifs, les médecins, lorsqu'il leur est fait référence de façon collective, y sont dépeints de façon tout à fait négative, de telle sorte que l'image méliorative qui est donnée du docteur Sachs tranche fortement sur cette toile de fond. Ainsi, quand ce dernier déclare: "Les médecins sont bouffis de suffisance et d'incompétence » (MS, p. 590), les autres narrateurs lui donnent raison, une secrétaire médicale affirmant: «tu ne me prends pas pour de la merde, comme le font la plupart de tes confrères » $(M S$, p. 225). Les exemples d'incompétence abondent. Un patient confie en effet : "J'avais peur qu'il me demande de m'allonger, la plupart des médecins ne savent pas que c'est pire. » $(M S$, p. 342) Ailleurs, la mère d'une fillette fait état d'un traitement - «des injections de 
globulines » $(M S$, p. 45) - qui a été appliqué à son neveu : «il paraît que ça leur fait du bien ». Le docteur Sachs accueille cette suggestion avec « une moue dubitative » : "Mmmmhh, ce n'est pas nécessaire. » Sa réputation se construit par le bouche à oreille :

Je viens parce qu'on m'a parlé de vous, il paraît que vous savez bien soigner l'asthme/la sinusite/les verrues/les migraines/la dépression/les rhumatismes/les furoncles/les personnes âgées et que vous êtes doux avec les enfants. (MS, p. 95)

À noter que le docteur Sachs avait déjà en tant qu'étudiant fait preuve d'une rare compétence, ainsi que le souligne son ancien professeur, le docteur Zimmermann: «Ça m’a exaspéré. L'externe savait mieux que le patron ce qu'avait la malade, ça la foutait mal.» (MS, p. 113) Parfois, la critique des autres médecins est implicite. Ainsi, quand un homme déclare : « Il me pique sans me faire mal, trouve la veine tout de suite, me fait l'injection très lentement» $(M S$, p. 343), le lecteur comprend qu'a contrario, les confrères du docteur Sachs n'ont pas les mêmes capacités.

« Les médecins mentent, non parce qu'ils ont peur de dire la vérité, mais parce-que-les-patients-préfèrent-ne-pas-laconnaître. On ne va tout de même pas les forcer! » (MS, p. 593), déclare avec ironie le docteur Sachs. Les propos qu'il prête à ses patients confirment ses accusations : "De toute manière, les médecins ils ne te disent jamais ce qu'ils pensent, ils te disent : C'est rien, on va vous arranger ça, vous avez bien fait de venir et, le temps que tu réalises, tu meurs sur la table d'opération » $(M S, 365)$. À l'inverse, un de ses malades affirme que le docteur Sachs, « c'est pas le genre à raconter des histoires » (MS, p. 562). Alors que «les docteurs ça ne prend pas toujours le temps d'écouter » $(M S$, p. 149), lui, en revanche, est apprécié de ses 
patients : « ils disent que tu les écoutes bien » (MS, p. 135), « Tu commentes, tu expliques. » (MS, p. 225)

Le docteur Sachs va jusqu'à proclamer que «tous les médecins sont des bourreaux» (MS, p. 594). "[J]amais», ajoute-t-il, « on ne m'a appris à soulager la douleur, ou à éviter qu'elle n'apparaisse. » (MS, p. 597) Mais si, comme le relève une pharmacienne, ses «confrères $\mathrm{n}[\mathrm{e}]$ demandent jamais [d']antalgiques [...], [prétendant] que la douleur c'est utile» (MS, p. 293), lui « ne laiss[e] jamais personne souffrir »: «Les mamans t'appellent Docteur Aspirine, les vieux t'appellent Docteur Soulagement. " Tandis que les médecins répètent à leurs malades : « je ne peux plus rien pour vous!» (MS, p. 234), Bruno répond à ses patients que "[q]uelle que soit la maladie, on peut toujours faire quelque chose » (MS, p. 237).

Le lecteur qui pourrait juger trop favorable et, partant, trop peu réaliste, le portrait que le roman brosse du docteur Sachs peut néanmoins trouver, ici ou là, quelques critiques que certains de ses patients lui adressent. Pourtant, à les examiner de près, ces critiques ne sont pas fondées et, par conséquent, derrière les prétendus défauts reprochés à Bruno, ce sont une fois de plus des qualités qui se font jour. Ainsi, un patient lance :

Je ne veux plus le voir: la dernière fois, il a refusé de me prescrire mon médicament contre le cholestérol. Il dit que ça ne sert à rien et que c'est dangereux. Mais enfin, si les médicaments faisaient plus de mal que de bien, les docteurs n'en prescriraient pas! [...] Non mais! Pour qui il se prend? Je sais quand même mieux que lui de quoi j'ai besoin. C'est qui, le malade? (MS, p. 216)

Ici, c'est en effet le malade qui, aveuglé à la fois par sa bêtise et sa prétention, condamne le savoir et l'honnêteté du docteur Sachs au nom de l'ignorance ou de l'irresponsabilité des 
confrères de celui-ci. De même, lorsqu'un autre patient confie : " Tu es très sympa et tu es très clair, mais je trouve que tu poses des questions un peu trop personnelles. Ma vie privée, après tout, ça n'a rien à voir avec ma santé » (MS, p. 207), c'est lui qui manifeste sa méconnaissance des relations existant entre les maladies, les activités ou les émotions.

C'est dire que, comparé à un milieu médical plutôt caricaturé, le docteur Sachs, loin d'apparaître comme un personnage authentique, renvoie davantage à une image idéalisée du médecin : "en dehors de toi, question médecine, [on] ne fait confiance à personne » $(M S$, p. 288), pourraient déclarer tous les narrateurs de La Maladie de Sachs. Face aux médecins qui se confondent avec le stéréotype de l'« agent de l'ennemi (praticien mercenaire, docte tueur à gages) » $(M S$, p. 150), le docteur Sachs, lui, «a toujours eu un faible pour les victimes. Ça l'émeut. » (MS, p. 323) Il est un médecin totalement différent des autres médecins, comme l'illustrent par exemple ces deux affirmations: " tous les docteurs ne sont pas comme vous » (MS, p. 236-7), «Ah, ben ça alors, c'est pas banal! Ça serait bien le premier! » (MS, p. 417) En outre, il ne se distingue pas seulement en tant que médecin, mais aussi en tant qu'« être humain ». La déclaration d'une de ses patientes en fait foi : «les hommes sont des salauds. [...] mais ce médecin, je dois reconnaître qu'il m'a vraiment tirée d'affaire » (MS, p. 440). En écho à une assertion du docteur Sachs - «La vie est un enfer » (MS, p. 379) - , une autre de ses patientes se fait une sombre image de la société tout entière :

le monde est gris, tout le monde est médiocre, les gens sont veules et cons. Tout le monde s'emmerde et passe son temps à haïr, les hommes à haïr les femmes, les femmes à haïr les hommes, les hommes à s'entre-tuer, les femmes à s'entre- 
déposséder. Tout le monde est nul et personne ne croit à l'amour. (MS, p. 474)

Cependant, de Bruno et de son amie, Pauline, elle dit : " ils sont mon seul soleil dans cette taule ». Tous les deux forment un couple hors du commun: «je n'avais jamais vu un homme embrasser une femme avec une telle tendresse, ni une femme fermer les yeux comme ça pour recevoir un baiser » $(M S$, p. 471).

C'est donc sur la vision critique et pessimiste d'une société et, à l'intérieur de celle-ci, du microcosme médical que se détache la figure en tous points positive du docteur Sachs. Or, cette dichotomie est la marque que, loin de représenter une image fidèle de la réalité, le réel du roman est une fiction qui obéit à la subjectivité de son narrateur. Ainsi, les déficiences inhérentes au monde médical sont en fait accentuées au travers du prisme déformant de l'esprit à la fois critique et pessimiste de Bruno Sachs, dont il est dit : «Tu as toujours été un drôle de type. » (MS, p. 125) La propension à la révolte constitue un des traits de sa personnalité : "Tu avais vingt ou vingt-deux ans et tu étais déjà en colère.» ( $M S$, p. 114) La présentation de La Maladie de Sachs par l'éditeur, en identifiant notamment le roman à un "pamphlet», établit un lien entre le texte et le caractère du narrateur :

À la fac, les étudiants l'appelaient l'inoxydable Sachs parce qu'il n'arrêtait pas de leur faire la morale [...]. Quelques copains disaient l'inox, ou l'intox, parce qu'il n'arrêtait pas de coller des pamphlets sur les murs de la fac de médecine, du genre "Ordre Médical, Ordre Nouveau?" ou "Nous sommes tous des médecins nazis". (MS, p. 317)

Le roman garde du reste l'empreinte de ce passé puisqu'il reproduit ce dernier tract (voir $M S$, p. 586-589). Son activisme 
n'ayant pas abouti, Bruno Sachs « est devenu un type plutôt sombre, plutôt taciturne » (MS, p. 318).

Or, ces prédispositions initiales ont été aggravées par la pratique de la médecine. Là aussi, le texte est éclairant. Au fil des années, le docteur Sachs, aux dires de sa secrétaire, devient « souvent silencieux, irritable » $(M S$, p. 136), « souvent triste et nerveux » (MS, p. 137); il a « l'air préoccupé » ( $M S$, p. 138). Une de ses amies est également le témoin de sa métamorphose : «Il $\mathrm{y}$ a comme de la colère dans son silence, comme de la haine dans ses paroles.» (MS, p. 309) C'est que "[l]a médecine», affirme le docteur Sachs, " est une maladie qui frappe tous les médecins » (MS, p. 655) et comme, en France, «les maladies, comme les syndromes, portent le nom des médecins qui les ont, sinon observés, du moins décrits pour la première fois » $(M S$, p. 466), cette maladie, dont il est atteint lui-même, devient la Maladie de Sachs. Celle-ci est provoquée par deux phénomènes principaux. Tout d'abord, il s'opère entre le malade et son médecin comme une réaction osmotique, les émotions négatives que celui-là nourrit envahissant le psychisme de celui-ci : le docteur Sachs est «malheureux d'avoir été témoin de tant de misère affective, de tant de haines rentrées, de tant de malentendus empilés » $(M S$, p. 228). Du fait qu'il est «bien trop sensible » (MS, p. 162), il lui est impossible de rester étranger aux petits et grands drames que vivent ses patients : « Tu comprends qu'[ils sont] dans la merde, et qu'[ils] te demande[nt] d'y mettre les mains. » (MS, p. 149) La seconde cause de la maladie des médecins tient aux limites du savoir médical : «On sait comment la maladie évolue. On sait à peu près soulager les symptômes. Mais on ne sait pas la guérir. » (MS, p. 233) Certains médecins échappent au découragement et à la haine de soi en faisant comme s'ils étaient réellement 
efficaces : "Il n'y a que des cinglés pour vouloir sauver la vie des gens, sans se rendre compte que c'est impossible. Ceux qui font semblant de croire le contraire sont des salauds. " (MS, p. 374) Bruno Sachs, lui, a parfaitement conscience de son impuissance.

De surcroît, cette impuissance à guérir lui a été confirmée dans des circonstances particulièrement tragiques, le docteur Sachs ayant été incapable d'éviter la mort à son père : «Non, je ne sais pas soigner. Si j'avais su, je l'aurais soigné, lui. Je l'aurais accompagné quand il est mort, mais je n'ai pas su. [...] Je ne l'ai pas soigné, je lui ai montré sa mort! » (MS, p. 513) En effet, la médecine s'est révélée en cette situation sous son véritable jour: dépourvue de moyens de lutte efficaces contre les maladies, mais riche en connaissances en ce qui concerne l'évolution des pathologies :

Quand mon père est tombé malade, et qu'on lui a annoncé de quoi il était atteint, j'étais tellement angoissé que je lui ai demandé de m'expliquer de quoi il s'agissait. C'était une maladie qu'il ne connaissait pas, parce qu'elle était rare, et très éloignée de sa spécialité. J'ai cherché un article sur le sujet. J'en ai trouvé un, qui venait d'être publié. Je le lui ai apporté. ( $M S$, p. 482)

Loin d'avoir pu guérir son père, voire simplement le soigner, le docteur Sachs a vu les insuffisances de la médecine rendre son rôle non seulement inutile, mais nocif : son intervention s'est limitée à apprendre avec précision à son père la façon dont celui-ci allait mourir. D'où un sentiment profond de culpabilité : «Mais je n'arrive pas à me défaire de cette idée que je n'ai pas vraiment le droit d'être vivant, moi, alors que mon père est mort. » (MS, p. 513) D'où la tentation du suicide : "J'ai plein de monde dans la salle d'attente, mais pas envie de bosser. Plutôt 
de me tirer une balle dans la tête. » (MS, p. 266) D'où, également, la violence de sa révolte contre l'institution médicale et la peinture qu'il est possible d'en discerner dans La Maladie de Sachs, subjective et, sur bien des points caricaturale et, partant, éloignée du réel. En définitive, le narrateur de cette fiction pourrait parfaitement s'approprier ce que Martin Winckler affirme dans Plumes d'Ange: «je ne raconte pas ici la réalité, je raconte ici ce que j'ai ressenti » (2003, p. 381).

\section{Autobiographie et fiction}

Ainsi, en dépit des différents procédés d'écriture qui peuvent, dans un premier temps, laisser penser que ce roman vise à décrire le réel et parvient à l'appréhender, il apparaît en fait que la part fictionnelle y est prédominante dans la mesure où l'univers romanesque reflète, en grande partie, le psychisme du narrateur. Cela posé, il reste à compléter - car la relation du texte avec le réel participe également de cette donnée l'analyse des liens existant entre le narrateur, le docteur Sachs, et l'auteur, Martin Winckler/Marc Zaffran, analyse qui a été esquissée au début de la présente étude.

Nombreux sont les éléments textuels qui militent en faveur de l'identité de l'un et de l'autre. Nous nous limiterons à quelques exemples significatifs. La «thèse de médecine imprimée à Alger en $1939 »(M S$, p. 661) dont est extrait le Serment d'Hippocrate reproduit à la page $17 \mathrm{du}$ roman est celle du père ${ }^{5}$ de l'auteur, Ange Zaffran, «Docteur en médecine en

5 L'information nous a été communiquée par M. Winckler (courriel du 14 avril 2007). 
$1939 »(2003$, p. 29). Comme le jeune Bruno (voir $M S$, p. 176), le jeune Marc est « plus littéraire que scientifique » (2003, p. 371). Toujours comme Bruno (voir $M S, 133$ ), il a tenu son journal sur des «cahiers" (2003, p.416) lorsqu'il était «médecin en formation » (2003, p. 390). L'anecdote (voir $M S$, p. 114) relatée par un professeur de médecine de Bruno correspond à une expérience vécue par Marc avec l'un des ses "patrons » (voir 2003, p. 396 et 397). Comme le docteur Sachs (voir MS, p. 482), le docteur Marc Zaffran a fait lire à son père malade un article donnant l'évolution de son affection (voir 2003, p. 447). La communication, intitulée «SOUFFRIR, SOIGNER, ÉCRIRE » $(M S$, p. 643), est une version remaniée de la communication intitulée «Soigner, écrire, la médecine selon Martin Winckler» et prononcée par Marc Zaffran au colloque «Le corps souffrant entre médecine et littérature », qu'il co-organisa en 1994 à Cerisy-La-Sallé6.

Ces fragments (auto)biographiques sont-ils en mesure d'orienter la fiction vers le réel? Comme d'autres écrivains - Le Clézio par exemple ${ }^{7}$-, Martin Winckler a en parallèle écrit des textes autobiographiques. Ainsi, Plumes d'Ange «contient des faits avérés sur la vie d'un homme » (2003, p. 23), mais, ajoute l'auteur, "c'est inévitablement une fiction, puisque je vais les inventer (dans les deux sens du terme : découvrir, imaginer) et les raconter». De même, dans Légendes, il souligne qu' «une autobiographie ne raconte pas la vérité, toute la vérité, rien que la vérité» (2002, p. 317), et que, par conséquent, «[1]es événements qualifiés de réels, d'établis, d'indiscutables [...] sont des fables» (2002, p.18). Si les textes autobiographiques

\footnotetext{
${ }^{6}$ Les actes de ce colloque ont paru dans Agora, no 34-35, 1995.

7 Voir notamment son roman Onitsha et l'autobiographie correspondante, L'Africain.
} 
n'entretiennent avec le réel que des liens distendus, les romans peuvent difficilement prétendre à en tisser de plus étroits. Pourtant, ni les uns ni les autres ne renoncent à la vérité. L'assertion que Martin Winckler place dans la bouche du héros des Cahiers Marcœur, une fiction inédite, «Tout est vrai, puisque je l'invente. Tout est faux, puisque je l'écris » (2002, p. 9), met l'accent sur le couple mensonge/vérité et, ce faisant, elle fait écho à la dynamique qu'Alain Robbe-Grillet (voir Milat, 2005, p. 91109) place au cœur de ses romans, puis de ce qu'il appelle la Nouvelle Autobiographie : «le mensonge, [...] c'est une question posée à la vérité » (1968). L'écrivain n'a pas pour vocation de représenter, de reproduire ce qu'il est convenu d'appeler le «réel»- sans qu'il soit possible de s'entendre sur la signification exacte de ce terme - , mais de créer son vrai réel.

\section{Bibliographie}

Butor, Michel. (1957), La Modification, Paris, Minuit.

LE CLÉzIO, J. M. G. (1991), Onitsha, Paris, Gallimard;

—. (2004), L'Africain, Paris, Mercure de France, coll. « Traits et Portraits ».

MiLAT, Christian. (2005), « Robbe-Grillet: autobiographie ou anthropographie? ", dans Roger-Michel ALLEMAND et C. Milat (dir.), Le «Nouveau Roman » en questions 5 : une "Nouvelle Autobiographie»?, Paris, Lettres modernes Minard, coll. « La Revue des Lettres modernes/L'Icosathèque $\left(20^{\text {th }}\right) »$, p. 91-109. 
RoBBE-GRILlET, Alain. (1968), « L'Express va plus loin avec Alain Robbe-Grillet », L'Express, $1^{\mathrm{er}}-7$ avril, p. 175.

WATZlaWick Paul, John WEAKLAND et Richard FISCH. (1975),

Changements : paradoxes et psychothérapie, Paris, Seuil.

WINCKLER, Martin. (1989), La Vacation, Paris, P.O.L;

—. ([1998] 2005), La Maladie de Sachs, Paris, Gallimard, coll. « Folio »;

-. (2002), Légendes, Paris, P.O.L;

—. (2003), Plumes d'Ange, Paris, P.O.L;

-. (2004), Les Trois Médecins, Paris, P.O.L.

\section{Résumé}

La réalité de la pratique médicale dont La Maladie de Sachs entend témoigner semble s'appuyer sur l'expérience du $\mathrm{Dr}$ Sachs comme sur celle de l'auteur, Martin Winckler, lui aussi médecin, celui-ci se confondant d'ailleurs à la fin du roman avec celui-là. Pourtant, il est permis de douter de la qualité de ce réel. Ainsi, le procédé consistant à faire intervenir, au milieu des rares narrations du Dr Sachs, une multitude de voix (celles des patients, de la mère, des amis, etc.), loin de renforcer l'effet de réel, participe à son altération, ces voix étant contaminées par le savoir et le vocabulaire médicaux du narrateur-auteur. De plus, ces voix épousent la vision de la médecine de Sachs/Winckler, vision largement pessimiste. Bref, au lieu de peindre le réel, le roman en crée une image subjective, celle que le Dr Sachs perçoit, notamment au travers de sa maladie, désespérance mêlée de révolte, toutes deux issues de l'impuissance cruellement ressentie par le jeune Dr Sachs face à la mort de son père. 


\begin{abstract}
La Maladie de Sachs bears witness to a reality of medical practice seemingly drawn from the experiences of both Dr. Sachs and the author. In fact, at the end of the novel, Martin Winckler, himself a physician, merges with his character. However, one might doubt the accuracy of this reality. The intervention, in the middle of Dr. Sachs' rare narratives, of a multitude of voices (those of his patients, his mother, his friends, etc.) instead of reinforcing this impression of reality, contributes to its deterioration because these voices are contaminated by the medical knowledge and vocabulary of the narrator-author. In addition, these voices share Sachs/Winckler's mostly pessimistic view of medicine. Instead of depicting the true reality, the novel creates a subjective image, based on what Dr. Sachs perceives, in part through his illness. This image arises out of the desperation and revolt flowing from the powerlessness cruelly felt by a young Dr. Sachs when confronted with his father's death.
\end{abstract}

